\title{
Association of Dietary Inflammatory Index (DII) and Depressive Disorders
}

\author{
Ruiqiang $\mathrm{Li}^{1, *}$ \\ Wenqiang Zhan ${ }^{2, *}$ \\ Xin Huang' \\ Zhan Liu' \\ Shuaishuai Lv' \\ Jiaqi Wang' \\ Luyao Liang' \\ Yuxia Ma'
}

'Department of Nutrition and Food Hygiene, School of Public Health, Hebei Medical University, Hebei Province Key Laboratory of Environment and Human Health, Shijiazhuang, People's Republic of China; ${ }^{2}$ School of Public Health, Shanghai Jiao Tong University School of Medicine, Shanghai, 200025, People's Republic of China

*These authors contributed equally to this work
Correspondence: Yuxia Ma

Department of Nutrition and Food Hygiene, School of Public Health, Hebei Medical University, Hebei Province Key Laboratory of Environment and Human Health, Shijiazhuang, People's Republic of China

Email mayuxia@hebmu.edu.cn

\begin{abstract}
A lot of evidence shows that inflammation is related to the development of depression. However, the heterogeneity of depression hinders efforts to understand, prevent and treat this disease. The purpose of this comprehensive review is to summarize the links between inflammation and the established core features of depression, which show more homogeneity than the syndrome itself: overreaction to negative information, changes in reward processing, and cognitive control decline, and somatic syndrome. For each core feature, we first briefly outline its relevance to depression and neurobiological basis, and then review the evidence to investigate the potential role of inflammation. We mainly focus on the discovery of the experimental paradigm of exogenous inflammation. We concluded that inflammation may play a role in overreaction to negative information, altered reward responses, and physical symptoms. There is less evidence to support the effect of inflammation on cognitive control by standard neuropsychological measures. Finally, we discussed the implications for future research and recommendations on how to test the role of inflammation in the pathogenesis of heterogeneous mental illness.
\end{abstract}

Keywords: dietary inflammatory index, depressive disorders, inflammation

\section{Introduction}

In recent decades, the prevalence of mental health disorders has been increasing and is a major public health problem. The current worldwide prevalence of depression is estimated at 350 million. ${ }^{1,2}$ According to the World Health Organization, more than a quarter of European adults have experienced psychological disorders. Data from the Global Burden of Disease Study highlights the significant contribution of mental health and depression to the global burden of non-communicable diseases, including years of disability. ${ }^{3}$ Therefore, it is important to determine new preventive measures or strategies to reduce the development of the disease. Multi-factor processes, most likely to involve biological, social, genetic, and environmental factors, contribute to the individual's mental health and well-being., ${ }^{4,5}$ There is increasing attention to the possible contribution of modifiable lifestyle behaviors (such as habitual dietary intake) to the development of common mental health disorders. In addition, the emerging field of nutritional psychiatry no longer focuses on specific nutrients or foods but turns its attention to the relationship between eating patterns and mental health. ${ }^{6,7}$

Healthy eating patterns, such as the Norwegian and Mediterranean diets, which are characterized by a high intake of fruits, vegetables, whole grains, fish, and lean meat, are associated with a lower likelihood of depression. ${ }^{8-10}$ On the contrary, it is 
reported that people with unhealthy or Western-style diets have an increased risk of depression, which is typically characterized by a large consumption of energy-dense, high-fat and high-sugar products, processed and red meat, refined grains, and alcohol. However, meta-analysis and systematic reviews have not yet provided confirmation, partly because of the limited number of studies. Eating patterns and diet quality are also related to wellbeing, anxiety, and stress, which suggests that the biological mechanisms that underpin the health-related diet go beyond depressive symptoms. Inflammation has been proposed as the basis of the mechanism linking diet to mental health. ${ }^{11,12}$ There is increasing evidence that depression is related to increased concentrations of pro-inflammatory cytokines. Although high heterogeneity was observed in earlier meta-analyses, a recent meta-analysis (MDD) of cytokines and chemokines in major depression confirmed that concentrations of tumor necrosis factor A (TNF-A) and interleukin-6 (IL-6), as well as other cytokines and chemokines, were significantly elevated in MDD patients. At present, relevant studies have reported that there is an association between the inflammatory potential of habitual diet and mental health, but the specific mechanism has not yet reached a consistent conclusion. ${ }^{13-15}$ Lucas et al reported Eating patterns related to circulating levels of C-reactive protein (CRP), IL-6, and TNF- $\alpha$ receptor 2 were determined, and their relationship with depression risk was examined among participants in the Nurses' Health Study. ${ }^{16-18}$ They reported a $30-40 \%$ increase in the risk of depression, depending on the definition, comparing the highest and lowest quintiles (that is, the most pro-inflammatory versus the most anti-inflammatory). ${ }^{19}$ In recent years, the Dietary Inflammatory Index (DII) has been developed to characterize an individual's continuous diet from maximum anti-inflammatory to proinflammatory. So far, DII has been associated with CRP, IL-6, and TNF-a. To date, only three studies have examined the relationship between DII and depression; all have reported that those with the most pro-inflammatory diets have an increased risk of depression. ${ }^{20}$

One candidate mechanism for motivation-related disorders in major depression is inflammation. Now, a large amount of literature shows that compared with the control group, some patients with depression have increased expression of inflammatory proteins and genes in peripheral tissues and cerebrospinal fluid (CSF), and increased peripheral blood acute phase proteins, chemokines, and adhesion molecules. A meta-analysis of these documents has determined that the most reliable inflammatory biomarkers for depression are the increase in peripheral blood inflammatory cytokines tumor necrosis factor (TNF) and interleukin (IL)- 6 and the increase in acute phase protein C-reactive protein. Finally, compared with the general population, in the list of common symptoms, nondepressed individuals with primary immune diseases showed a significantly higher incidence of anhedonia symptoms. ${ }^{21-23}$

Patients with depression show a wide range of clinical manifestations, including cognitive and autonomic symptoms. Importantly, these symptoms may differ in their biological causes, and deconstructing depression into specific symptoms may provide valuable insights into underlying neurobiology. A study has conducted a narrative review of the existing literature (21 studies) to clarify whether the link between depression and inflammation has specific symptoms. Overall, there is evidence that there is an association between neurovegetative symptoms of depression and inflammation, independent of cognitive symptoms. This is not the case with cognitive symptoms and inflammation. There is also some evidence that there are gender differences in the direction of the relationship between depression and inflammation. ${ }^{24}$ At the same time, a meta-analysis of 22 studies showed that CRP and IL-6 inflammatory cytokine levels are significantly positively correlated with the risk of concurrent depression. At the same time, inflammation (measured by CRP or IL-6) can predict future depression. This suggests that there may be a two-way relationship between depression and pro-inflammatory states. ${ }^{25}$ Another review identified the cross-sectional and longitudinal associations of two inflammatory markers, C-reactive protein (CRP) and interleukin 6 (IL-6), and depression in the elderly. The results show that there is a cross-sectional and longitudinal relationship between CRP and IL-6 and depression in the elderly. In longitudinal studies, inflammation causes depression, rather than inflammation. ${ }^{26}$

Elevated biomarkers of systemic inflammation have been reported in individuals with cognitive decline; however, most of the literature involves cross-sectional analyses with mixed results. A study investigating the etiology of this association conducted a meta-analysis of prospective studies examining the association between baseline interleukin-6 (IL-6), an established marker of peripheral inflammation, and the risk of cognitive decline at follow-up in adults without dementia. At the same time, mindfulness-based intervention (MBI) has a positive impact on the mental health of different 
populations. One study examined the effects of MBI on a psychiatric biomarker used to summarize the effects of low-grade inflammation. The results showed that MBI showed a low but significant effect on health outcomes associated with low-grade inflammatory biomarkers. ${ }^{22}$

\section{Other Diet-Related Indexes and Depression The Dietary Approach to Stop Hypertension (DASH)}

The Dietary Approach to Stopping Hypertension (DASH) is based on seven components, with the four highest intakes considered ideal (vegetables, fruits, whole grains, and legumes) and three components, with avoidance or minimum intakes, considered ideal (conversion and red meat, sweet drinks, and sodium). The minimum value of each section is 1 , the maximum value is 5 , and the middle value is graded proportionately. ${ }^{27,28}$ The scores for all components are added up to a DASH total score ranging from 5 to 40, with higher scores indicating a healthier diet. $^{29}$

There is increasing evidence that there is a link between depression and high blood pressure. Unhealthy lifestyle habits, such as high-energy intake and poor diet, smoking and drinking, and lack of physical exercise, are all risk factors for depression and high blood pressure. In addition, depression and hypertension are related to the imbalance of redox activity signaling and increased immune-inflammatory response. ${ }^{30,31}$ These diseases may lead to excessive activation of the hypothalamic-pituitaryadrenal axis, leading to increased secretion of corticotropin-releasing factor, corticotropin, and cortisol. These known risk factors are increased blood pressure and depressive symptoms. Oxidative stress and mild inflammation may also cause autonomic nervous system dysfunction, including increased sympathetic nerve activity and decreased parasympathetic nerve activity, and promote high blood pressure and depression. ${ }^{32}$ In addition, due to the role of endothelial cells in the production of brainderived neurotrophic factors, endothelial dysfunction is considered a risk factor for depression. Endothelial dysfunction is also a risk factor for high blood pressure because it may lead to an increase in blood pressure and the vascular endothelium may acquire pro-inflammatory properties. $^{33}$

The Diet to Stop High Blood Pressure (DASH) diet plan, which includes following a diet rich in fruits, vegetables, and low-fat dairy products, as well as low in SFA and red meat, is effective in reducing inflammatory markers and cardiovascular risk factors, including blood pressure And blood lipids. In addition, it has been reported that there is a negative correlation between adherence to a DASH diet and diabetes or CVD. Since hypertension and depression have many common risk factors, and because of the potential benefits of the DASH diet for diabetes, CVD, and low-grade inflammation, it is determined that the dietary treatment of hypertension may be related to the risk of depression. ${ }^{34,35}$

Adhering to the DASH diet can not only reduce the risk of CRC and CRA in men and women but also reduce the risk of renal function decline and all-cause mortality. ${ }^{36}$ There is evidence that the protective properties of the DASH diet may be partly due to its inverse relationship with visceral obesity. ${ }^{37}$ Some studies have provided evidence that the Dietary Approaches to Stop Hypertension (DASH) diet is associated with reduced levels of oxidative stress and inflammation, and may provide effective means for chronic disease prevention. ${ }^{38}$ Compared with the conventional diet, adherence to the DASH diet can effectively improve the circulating serum inflammatory biomarkers in adults and promote the formation of a systemic antiinflammatory environment, at the same time, it can also reduce the risk of aggregate metabolism in elderly women. Consequently, it may be a valuable strategy to inhibit the inflammatory process. ${ }^{39,40}$ DASH diet can significantly increase GSH and reduce MDA levels. In addition, there is a trend to improve TAC, $\mathrm{NO}$, and $\mathrm{f} 2$-is prostaglandins by sticking to the DASH diet. ${ }^{41}$ Intake of the DASH diet for 8 weeks in NAFLD patients has significant effects on body weight, BMI, ALT, ALP, triglycerides, insulin metabolism markers, inflammation markers, glutathione, and malondialdehyde. ${ }^{42}$ Whilst, DASH diet in pregnant women with GDM has a beneficial effect on FPG, serum insulin levels, HOMA-IR score, plasma TAC, and total GSH levels. ${ }^{43}$

\section{The Alternative Healthy Eating Index}

The Alternative Healthy Eating Index 2010 (AHEI-2010) is based on 11 ingredients, of which six are considered ideal for maximum intake (vegetables, fruits, whole grains, nuts and legumes, long-chain omega-3 fats (DHA and EPA), and polyunsaturated fatty acids) and one is considered ideal for moderate intake (alcohol). Four ingredients to avoid or minimize are considered ideal (sugarsweetened beverages and fruit juices, red and processed 
meats, trans fats, and sodium). The minimum value of each section is 0 , the maximum value is 10 , and the middle value is graded proportionately. The scores for all the components are added to produce an aHEI-2010 overall score, which ranges from 0 to 110 , with a higher score indicating a healthier diet. ${ }^{44,45}$

Prospective studies have shown that, as measured by the Alternative Healthy Eating Index (AHEI), adherence to a healthy eating pattern is associated with a lower risk of recurrence of depressive symptoms in women, but not in men. This favorable association is attributed to the components of AHEI, including vegetables, fruits, and the ratio of PUFA/SFA. In addition, it is reported that according to the Healthy Eating Index (HEI)-2005 measurement, African Americans and white adults living in Baltimore have a higher quality of their diet and fewer symptoms of depression. ${ }^{46,47}$ Similar findings have been reported in the National Health and Nutrition Examination Survey, where a higher HEI is associated with a lower risk of depression in adults. The results of the meta-analysis of observational studies also revealed a significant negative correlation between healthy eating patterns (determined by factor analysis) and depression risk. ${ }^{48,49}$

Several possible mechanisms may explain the negative correlation between AHEI-2010 and depression. This association is caused by the cumulative effect of all the components of AHEI-2010, not by individual nutrients or food groups. The high content of folic acid, B vitamins, and antioxidants in a healthy diet may reduce the damage of oxidative stress to neurons. Due to the relationship between high levels of inflammatory biomarkers and depressive symptoms, the anti-inflammatory properties of the foods contained in AHEI have been shown to reduce the concentration of monoamines. In addition, the high levels of PUFA and n-3 fatty acids present in oily fish and other components of AHEI are other possible mechanisms. ${ }^{50-52}$

\section{The Relative Mediterranean Diet Score}

The Relative Mediterranean Diet Score (rMED) described by Buckland et al estimates compliance with MD and is based on 9 "ideal" or "bad" dietary components. In terms of health, the "ideal" ingredients include grains, vegetables (excluding potatoes), beans, fruits and nuts, fresh fish, olive oil, and moderate alcohol consumption. In turn, undesirable ingredients include meat and dairy products. Each ingredient (except alcohol) is expressed in grams per $1000 \mathrm{kcal}$ per day and is divided into gender-specific tertiles. Values between 0 and 2 are assigned to each tertile of the "ideal" component. ${ }^{53,54}$ For "unwanted" ingredients, the scores are reversed. For alcohol consumption, if the alcohol consumption is in the range of 5-25 g/day for women and 10-50 g/day for men, 2 points are allocated; otherwise, it is recorded as 0 points. rMED is the sum of all 9 components. The total score ranges from 0 points (non-compliance) to 18 points (complete compliance). ${ }^{55}$

MD is characterized by high consumption of olive oil, whole grains, and plant food, low in saturated fat and sugar intake, and moderate consumption of fish, dairy products, and red wine, is considered to be one of the best models of a healthy diet because it has several cardiovascular diseases, cancer, diabetes, metabolic syndrome, inflammation and oxidative stress, and the beneficial effects of overall mortality rates. In particular, recent evidence suggests that inflammation and oxidative stress may constitute potential pathways for a potential link between diet and depression. This is supported by several studies that report a significant association between the inflammatory potential of diet and depression or depressive symptoms. $^{56,57}$

Many possible approaches have been proposed to explain the link between diet and depression, including inflammation, oxidative stress, and regulation of neurotransmitter synthesis. Epidemiological studies report a negative correlation between MD compliance and inflammation and oxidative stress marker levels. Specifically, inflammation is the result of an imbalance between pro-inflammatory and anti-inflammatory mediators. In the brain, pro-inflammatory cytokines, such as IL6 and TNF- $\alpha$, affect almost all pathways involved in the pathophysiology of depression, such as changes in neurotransmitter expression, neuroendocrine function, and synaptic plasticity. ${ }^{58,59}$

Certain Mediterranean diet components may affect preclinical vascular changes through potential new mechanisms. These changes may be related to cardiovascular risk by reducing endothelial oxidative stress, reducing inflammation, and enhancing endothelial function. ${ }^{60}$ Furthermore, greater adherence to the Mediterranean diet, especially its fish composition is related to lower CRP blood concentration. ${ }^{61}$ MedDiet has similar effects on inflammation in men and women. In addition, the variability of MedDiet's anti-inflammatory effects may be partly due to the individual's overall inflammatory state; however, this observation seems to be more specific to men. ${ }^{62}$ In addition, the understanding of how nutrition 
affects the prevention and/or treatment of neurodegenerative diseases has also been greatly developed in recent years. The protective effect of the Mediterranean diet (MD) in preventing neurodegenerative diseases has been reported in many studies. The distinctive feature of the Mediterranean diet is the moderate intake of red wine and extra virgin olive oil, both of which are rich in polyphenolic compounds, such as resveratrol, oleuropein, hydroxytyrosol, and its derivatives. In vitro studies have shown They have neuroprotective properties, including antiinflammatory effects on microglia. ${ }^{63}$

\section{Dietary Inflammatory Index (DII) Overview of DII}

DII is an inflammatory index developed and verified by a researcher at the University of South Carolina in Columbia to evaluate the inflammatory potential of an individual's diet to understand the inflammatory impact of diet on human health. The purpose of creating the inflammation index is to provide a tool to classify an individual's diet from the most anti-inflammatory to the most pro-inflammatory continuity. The original DII was derived from the results of articles evaluating the effects of specific foods and ingredients on specific inflammatory markers published from 1950 to 2007. Each food and ingredient was assigned a score and multiplied by the actual food intake parameters of the individual to produce a The overall score summarizes a person's diet from the maximum anti-inflammatory to the maximum proinflammatory degree. Specific markers are IL-1 $\beta$, IL-4, IL-6, IL-10, TNF- $\alpha$ and CRP. In 2014, South Carolina researchers improved the original DII scoring for peerreviewed journals updated from 2007 to 2010; the improved scoring system has been applied to 45 food parameters, including whole foods, nutrition, and other biologically active compounds, from all over the world Eleven food consumption data sets were determined, and the entire diet was considered, not just individual nutrition or food, and a comprehensive database was established to represent various diets of different populations in different regions of the world, which can be applied globally. The DII includes micronutrients, micronutrients, and other common dietary components, such as flavonoids and caffeine. It is a new tool for evaluating the potential of dietary inflammation and can be applied to any population that has collected dietary data. DII can be obtained through 24hour diet recall interviews or food record data. Food parameters can increase or decrease inflammatory potential through pro-inflammatory and anti-inflammatory markers; according to the influence of food parameters on inflammation, significantly increase IL- $1 \beta$, IL-6, TNF- $\alpha$ or CRP, or decrease IL-4 or IL-10 Give "+1", it is a proinflammatory effect; significantly reduce IL-1 $\beta$, IL-6, TNF- $\alpha$ or CRP, or increase the "-1" given by IL-4 or IL10 , which is an anti-inflammatory effect; if food If the parameter does not produce any significant changes in inflammation markers, " 0 " is assigned. ${ }^{64-66}$

\section{Calculation of DII}

The steps to establish a personal DII are as follows. (1) The calculation of DII is based on dietary intake data and then linked to a representative database, which provides a reliable mean and standard deviation for 45 food parameters, and calculates the Z score of each person's food parameter, to balance the right bias Influence, the $Z$ value is converted to the central percentile. (2) The calculation of food-specific inflammation score is first weighted according to the research design and research type. These weighted valuesare used to calculate the pro-inflammatory component of each food parameter: a. Divide the number of weighted pro-inflammatory articles by the total number of weighted articles, b. Subtract the anti-inflammatory score from the pro-inflammatory score, and choose 236 as the critical point, that is, the median of the total number of weighted articles of all food parameters, to represent an optimal and robust literature library, all with weighted articles $\geq 236$ Food parameters are assigned to the full value of the score. Adjust the foods and ingredients with weighted foods $<236$ as follows: divide the weighted foods by 236 ; then multiply the scores by the food parameter-specific original inflammatory effect score to obtain the food parameter-specific overall inflammatory effect score. (3)Multiply the central percentile value of each food parameter by its respective "overall food parameter specific inflammatory effect score" to obtain the "food parameter specific DII score". (4) Add up all "food parameter-specific DII scores" to obtain personal "DII scores". ${ }^{67}$

\section{Correlation Between DII and Inflammation}

Chronic low-grade inflammation has complex and multifocal endogenous and exogenous causes, and diet has always been considered an important factor in regulating inflammation. A healthy diet (such as a Mediterranean 
diet, rich in fruits and vegetables) is usually associated with lower levels of inflammation, and a Western diet (such as high fat and simple carbohydrates) is associated with higher levels of inflammatory markers, which can cause inflammation in the immune system. ${ }^{68,69}$ Belongs to the inflammatory diet. Studies have shown that the Western diet can change the intestinal microbial ecosystem and promote the occurrence and development of chronic inflammation. A study on cardiovascular risk showed that complement components C3, CPR, IL-6, TNF- $\alpha$, WBC, and neutrophil/lymphocyte ratio (NLR) are all increased in people with higher DII scores, and NLR is a sign of systemic inflammation. A meta-analysis on the relationship between DII and the risk of prostate cancer showed that a pro-inflammatory diet can lead to increased cell proliferation, inflammation, and oxidative stress, leading to prostate cancer. The possible mechanism is the promotion of foods such as butter, meat, and candy. An inflammatory diet increases systemic inflammation and causes insulin resistance, leading to an increase in insulin levels. Insulin levels play a role in the occurrence of prostate cancer by stimulating cell proliferation and apoptosis. $^{70,71}$ A study in the United States showed that adherence to a pro-inflammatory diet including cholesterol and saturated fat has independent positive correlations with inflammation markers of white blood cells, IL-6, and homocysteine, and intake of high sugar, saturated fat, and other pro-inflammatory foods It promotes inflammation, which may increase the risk of various chronic diseases. On the other hand, the intake of the Mediterranean diet (including whole grains, fish, fruits and green vegetables, olive oil, etc.) is associated with lower levels of inflammation and is an anti-inflammatory diet. Adherence to the Mediterranean diet is associated with lower CRP, platelets, and WBC has antiinflammatory effects and has a protective effect on cardiovascular diseases. ${ }^{72,73}$

\section{Influencing Factors of DII Gender and DII}

Mental health disorders are more common in women than in men. According to reports, there are gender differences in depression and anxiety, including the age of onset and duration of symptoms, social adaptation, and long-term outcome. Current work shows that there are associations between the potential for dietary inflammation and depressive symptoms, anxiety, and mental health, and these associations are particularly evident in female participants.
Although some earlier studies have also reported associations between female depression risk, these are femaleonly cohorts, and the dietary inflammation potential is determined by two different methods (dietary inflammation pattern and DII). The examination of the Nurses' Health Research showed that by definition, comparing the highest and lowest quintiles of dietary inflammation patterns (that is, the most pro-inflammatory versus the most anti-inflammatory), the risk of depression increased by $30-40 \%{ }^{74,75}$ The Australian Longitudinal Study of Women's Health $(n=6438)$ conducted a 12-year followup of middle-aged women and found that people with diet in the top quartile of DII had a $20 \%$ lower risk of depression than those in the lowest quartile of DII. To date, only two other studies have investigated the relationship between DII scores and the risk of depression in both sexes. ${ }^{76}$ In a Spanish cohort study of college graduates $(n=15,093)$, male and female participants reported similar increases in depression risk (OR 1.53 and 1.46, respectively). Consistent with our findings are the latest data from the Whitehall II study, which also revealed a sexspecific association between DII and recurrence of depressive symptoms. ${ }^{77}$ In a longitudinal analysis of middle-aged men and women (average follow-up age of 60 years), women with the highest DII scores (ie, most proinflammatory diets) were almost 3 times more likely to develop recurrent depressive symptoms, even after adjustment After a wide range of confounding factors. No association was observed among male study participants. Compared with the Mitchelstown and Whitehall II cohorts (average age of about 60 years), the Spanish cohort (average age of one-fifth of the DII is 36-40 years) may at least partly explain this difference in reporting specific gender relations Time. Further research is necessary to unravel the relationship between gender diet and depression. ${ }^{78,79}$

\section{$\mathrm{BMI}$ and DII}

Several studies have assessed the relationship between diet and the inflammatory potential of overweight or obesity. Higher DII scores indicate a diet that is more conducive to inflammation and are associated with higher BMI, waist circumference, waist-to-height ratio, and increased risk of cardiovascular disease and cancer. A cross-sectional study of the University of Minas Gerais cohort also showed that the prevalence of obesity increased with the DII score. In addition, a longitudinal study of a Mediterranean cohort also showed that higher DII scores were associated with increased annual mean weight change and incidence of 
overweight and obesity at 10 years of follow-up. Our findings are consistent with these previous studies that higher DII scores are associated with an increased risk of obesity, while lower DII scores are associated with a lower risk of obesity. ${ }^{80-82}$

A large cross-sectional study showed that in individuals with high cardiovascular risk, there is a direct correlation between DII and BMI and abdominal obesity. A recent meta-analysis of 12 prospective studies reported that subjects with the highest DII quartile had higher BMI levels. A meta-analysis of another observational study showed that there is a significant positive correlation between DII and obesity and BMI. On the other hand, some previous studies have shown that DII is negatively correlated with obesity, or has nothing to do with body weight, BMI, fat mass, and abdominal obesity. ${ }^{83}$ These controversies may be partly due to differences in the study population, metabolic disorders, BMI cut-off values, age, gender, and socioeconomic status, dietary supplements, and the number of foods and nutrients included in the DII calculation. It may also be related to reported deviations that are known to vary with weight status. ${ }^{81,84}$

DII provides a quantitative assessment of the potential for dietary inflammation. Current research results show that, compared with obese individuals, a normal-weight diet is more pro-inflammatory and manifests as a higher DII score. However, E-DII did not differ significantly among the three study groups. As mentioned earlier, this may be due to differences in total energy intake using FFQ, which are taken into account when scoring E-DII or energy-related reporting deviations. ${ }^{85,86} \mathrm{~A}$ follow-up cohort study showed that there is a direct link between DII and annual weight gain and overweight and obesity in normal-weight individuals. There seems to be a two-way relationship between obesity and inflammation. Body fat mass induces inflammation, which can lead to obesity and weight gain. In addition, a positive correlation was observed between the DII score and circulating leptin (as a pro-inflammatory fat-derived peptide), which underscores the potential role of dietary inflammatory features on adipose tissue inflammation. Chronic inflammation is one of the key mechanisms explaining obesity-related complications. $^{87,88}$

In the field of obesity, BMI is the most common and convenient obesity classification index. The significance of using BMI is far-reaching because it can give an accurate cut-off point to define the number of people with different weights, and then tell us the prevalence of obesity. Obesity is defined as the excessive accumulation of body fat, and this excess fat is usually considered an indicator of poor health, which in turn constitutes a series of risk factors for diseases, including diabetes, ischemic heart disease, hyperlipidemia, Sleep apnea, arthritis, and others. ${ }^{89}$

Adipose tissue is not only a storage organ for triglycerides, but also studies have shown the role of white adipose tissue as a producer of certain biologically active substances called adipokines. ${ }^{90}$

In mammals, white adipose tissue (WAT) stores and releases lipids, while brown adipose tissue (BAT) oxidizes lipids to promote thermogenesis. In obese individuals, WAT undergoes profound changes; it enlarges, becomes dysfunctional, and develops into a state of low-grade inflammation. $^{91}$ Adipose tissue is the source of proinflammatory activity, so it is called obesity-related inflammatory activity. ${ }^{92}$ Withal, adipose tissue itself is an endocrine organ that can secrete a variety of body fluid mediators, such as adipokines, which can induce or reduce inflammation and oxidative stress. ${ }^{93}$ Some studies have provided evidence that rodent models have demonstrated that fat inflammation pathways play an important role in diet and obesity-related metabolic disorders, and mRNA microarrays and secretion profiles of human adipose tissue and fat cells have identified similar inflammatory pathways in the obesity Inflammation pathway activated by endotoxin. $^{94}$

\section{Age and DII}

Studies have shown that there is a correlation between dietary inflammatory index and age, and aging is also closely related to elevated systemic inflammation markers. It is important to pay attention to how to measure these indicators in the context of dietary intervention. The levels of acute-phase response factor c-reactive protein (CRP), interleukin-6 (IL-6), and tumor necrosis factor (TNF) in the blood of the elderly tend to increase during the aging process. In the National Health and Nutrition Examination Survey (NHANES III), chronically elevated protein in the acute phase is a risk indicator of high mortality. The mortality rate of patients with elevated serum CRP over the age of 60 is 2.7 times higher than that of patients below this threshold $(0.30 \mathrm{mg} / \mathrm{dl}){ }^{95,96}$

Chronic inflammation is a persistent, low-level nonspecific body inflammation state. Studies have shown that chronic inflammation is related to cancer, diabetes, and cardiovascular diseases. ${ }^{97}$ In addition, studies have shown that the occurrence of old age frailty is 
accompanied by changes in an inflammatory state. Inflammatory factors induce cell senescence through oxidative stress, cell apoptosis, cell cycle arrest, and other pathways, causing tissue, organ, and system damage, which greatly increases the risk of debilitating or agerelated chronic diseases. ${ }^{98}$ A large number of studies have proved that diet plays an important regulatory role in chronic inflammation. Different dietary patterns and dietary ingredients will produce different inflammatory effects. Aging is also a contributing factor to inflammation caused by diet. The underlying mechanism remains to be studied, but it can be simply assumed that old age is related to a more unhealthy and unbalanced diet, which may lead to higher DII scores in the elderly population. Human aging is accompanied by changes that may impair food acquisition, digestion, and metabolism. Many conditions, such as disability, acute and chronic diseases, may affect the nutritional status of elderly patients. There is convincing evidence that diet changes with age, which may promote inflammation, which may help increase or maintain obesity, especially abdominal obesity, in most people who are overweight or obese..$^{99-101}$

\section{Possible Mechanism}

\section{Mechanisms Linking Inflammation and Depression}

Inflammation is a key component of the innate immune system's ability to clear infections and repair injured tissues. Inflammation is caused by the release of pro-inflammatory cytokines from innate immune cells. In addition to the effects on the periphery, cytokines can also communicate with the brain and cause a series of emotional, cognitive, and behavioral changes collectively referred to as "disease behavior." 102,103 Of particular relevance to this review is that peripheral inflammation has been shown to induce depression-like behaviors in animal models, including decreased exploratory, novelty, and social behavior, decreased food intake; and sleep disorders. These disease behaviors are considered an adaptive response designed to reduce the spread of infection and promote healing. However, long-term inflammatory signaling, such as when the inflammatory response is maintained by continuous psychosocial stress, may have adverse effects, including the risk of depression and other mental illnesses. ${ }^{104}$

Research conducted in the past few decades has clarified the mechanism by which peripheral inflammation can enter the brain to affect neural processes related to depression, including neuroplasticity, neurotransmitter systems, and neuroendocrine functions. For example, inflammatory cytokines can alter neuroplasticity by reducing the expression of the brain-derived neuroprotective hormone BDNF. Inflammation can also cause changes in the dopaminergic system, which is related to depression. A single injection of high doses of LPS $(5 \mathrm{mg} / \mathrm{kg}$ ) can cause the degeneration of the dopaminergic system in the brain, especially in the substantia nigra and striatum. ${ }^{105-107}$ In addition, the use of IFN- $\alpha$ for chronic treatment in nonhuman primates leads to a decrease in the synthesis and availability of dopamine in the striatum, which is related to a reduction in sucrose consumption that depends on effort. A large number of experimental studies conducted in rodents have shown that the release of dopamine and the reduction of dopamine receptor binding are closely related to the defects of reward motivation behavior in depression. $^{108}$

In addition to the effect on dopamine, inflammation can also affect the serotonin system through changes in tryptophan metabolism. Under normal, healthy conditions, approximately $5 \%$ of tryptophan is metabolized to serotonin, and $95 \%$ of tryptophan is metabolized through the kynurenine pathway. The metabolism of tryptophan through the kynurenine pathway can be converted into neurotoxic 3-hydroxykynurenine (OHK) and quinolinic acid (QUIN) or neuroprotective kynurenine. ${ }^{109,110}$ The way tryptophan is metabolized depends on the presence of indoleamine-2,3-dioxygenase (IDO). In preclinical models, the use of LPS to activate inflammation increases the activity of IDO, thereby assigning more tryptophan metabolism to the kynurenine pathway instead of serotonin synthesis, ultimately leading to serotonin deficiency. In preclinical models, LPS-induced depressive behavior is mediated by IDO activation. It is worth noting that inflammation and IDO both affect glutamate metabolism, which may also play a role in depression. ${ }^{111}$

In addition, inflammation has a powerful effect on the neuroendocrine system. Inflammatory cytokines may indirectly up-regulate glucocorticoids in the body by interfering with the functional ability of glucocorticoid receptors, leading to the continuous synthesis of corticotropin-releasing hormone $(\mathrm{CRH})$ and the continuous activation of the HPA axis, which have long been associated with the pathogenesis of depression-related. This process can be self-sustaining because long-term elevated glucocorticoids can stimulate the production of neuroinflammation by activating microglia (resident immune cells in the brain). ${ }^{112}$ 


\section{Related Mechanisms of DII and Inflammation}

Diet affects inflammation, but there are currently few studies on how diet affects inflammation markers. One theory is that a pro-inflammatory diet can increase the levels of inflammatory cytokines by affecting oxidative stress and immune mechanisms. Related studies have shown that macrophages will produce free radicals and release them into tissues after ingesting a proinflammatory diet. Free radicals promote cell oxidative stress. Excess free radicals are usually associated with increased inflammation, that is, pro-inflammatory diets can cause inflammation in the blood. The release of sex factors is enhanced, causing inflammation. Research by Barrera et al showed that Western diet can cause postprandial hyperglycemia and hyperlipidemia, and through non-enzymatic glycosylation and glucose-induced NADH: NAD ratio imbalance mechanism to generate reactive oxygen species to increase inflammation and oxidative stress and mediate Expression of inflammatory factors CRP, IL-6, IL-1 $\beta$. Increasingly, the diet is the main medium for the function of the microbiota of the gastrointestinal tract. ${ }^{113,114}$ A German study showed that in mice fed a pro-inflammatory diet, the integrity of the intestinal immune cell barrier can be disturbed or lost, that is, through different mechanisms to cause intestinal cytotoxic effects, inhibit adenosine monophosphateactivated protein kinase, and Causes the production of mitochondrial reactive oxygen species, activates the mouse NLRP3 inflammasome, and mediates the release of IL- $1 \beta$, that is, diet can directly and indirectly (through the intestinal microbiota) provide a rich source of biologically active substances, and produce local and systemic effects on immune function. Influence and mediate the development of inflammatory response. At the same time, the anti-inflammatory diet, the Mediterranean diet, can reduce the release of pro-inflammatory factors by reducing the proportion of Gram-negative bacteria in the intestine, improving the intestinal barrier function, and reducing endotoxins, thereby reducing metabolic endotoxemia, inflammation, and The subsequent occurrence of metabolic diseases. ${ }^{115,116}$

\section{Potential Mechanism Between DII and Depression}

The DII/E-DII is a summary measure for assessing the inflammatory potential of the diet. The construct validity of the DII and E-DII has been determined against inflammatory biomarkers in several different populations. Overall, these validated results support the notion that diet plays an essential role in modifying inflammation. Diets can be either proinflammatory or antiinflammatory, depending on the hormonal responses they generate. ${ }^{117}$ A pro-inflammatory diet may increase the chronic, persistent activation of the immune system, which leads to low-grade inflammation. Activation of immune cells, especially polymorphonuclear leukocytes, leads to overproduction of reactive oxygen species (ROS) resulting in oxidative stress. It is not well understood how oxidative stress leads to the development of depression. However, the most likely hypothesis is that the brain neuronal cells are vulnerable to oxidative stress due to their requirement of higher oxygen consumption and consequent generation of ROS, as well as a relatively weak antioxidant defense. ROS activates inflammasomes such as NLRP3 (NOD-, LRR and pyrin domain-containing protein 3), a cytoplasmic protein complex that modulates innate immune function by activating caspase-1, which increases pro-inflammatory cytokines such as IL1b. ${ }^{118,119}$ ROS also can regulate inflammatory processes by activation of transcription factors, including nuclear factor-kappa B (NF-kb) and activator protein-1 (AP-1) that lead to increased expression of proinflammatory cytokines. Conversely, these cytokines either: 1) stimulate indoleamine 2,3-dioxygenase (IDO) to convert tryptophan to kynurenine which is transformed into the neurotoxic quinolinic acid; or 2) exert an effect on the hypothalamic-pituitary-adrenal (HPA) axis which is linked to a reduction in hippocampal volumes, impaired neuronal plasticity, and decreased neurochemical functioning, resulting in DepS. ${ }^{120,121}$

Another potential mechanism through which diet may influence DepS includes the brain-gut-microbiota axis, a bilateral communication network between the intestine and brain. The intestinal microbiota and diet play an essential role in these gut-brain interactions and be involved in the pathogenesis of psychiatric disorders, including depression. Depression is associated with an altered gut microbiota composition, richness, and diversity. ${ }^{122,123}$ First, the neurotransmitter serotonin may have antidepressant and anxiolytic effects. Tryptophan, the main precursor of serotonin, is predominantly produced $(>90 \%)$ by the gut microbiota. It is evident that the consumption of probiotics, specifically, Lactobacillus spp. and Bifidobacterium spp., affect mood by influencing serotonin levels. Second, in some preclinical studies in mice, consumption of a typical Western diet (high in animal protein and fat, low in fiber) led to a marked decrease in 
the numbers of total bacteria and a proportional reduction of beneficial Bifidobacterium or Eubacterium species leading to diet-induced dysbiosis. This phenomenon results in increased permeability of the intestinal mucosa, also known as "leaky gut", which may increase the immune response and chronic neuroinflammation. This, in turn, stimulates pro-inflammatory cytokine production, which occurs when bacterial components such as lipopolysaccharides from the bacterial cell wall binding to circulating macrophages or monocytes. ${ }^{124-126}$

\section{Conclusion}

A growing body of evidence supports the potential use of dietary interventions as adjunctive treatments for mental disorders. This review identified many ways that diet may affect mental health. These include pathways that regulate inflammation, oxidative stress, mitochondrial dysfunction, gut microbiota, tryptophan-kynurenine metabolism, HPA axis, neurogenesis and BDNF, epigenetics, and obesity (Figure 1). However, we do acknowledge that there are many other potential mechanisms involved in the pathophysiology of depression. These mechanisms are not mentioned in this article, but all of them can further reduce the risk of depression through dietary interventions, such as dietary effects on leptin, adiponectin, and mitochondrial biosynthesis. Research highlights the potential of restricting pro-inflammatory diets and/or supporting antiinflammatory diets in reducing the risk of depression. However, more prospective longitudinal studies and

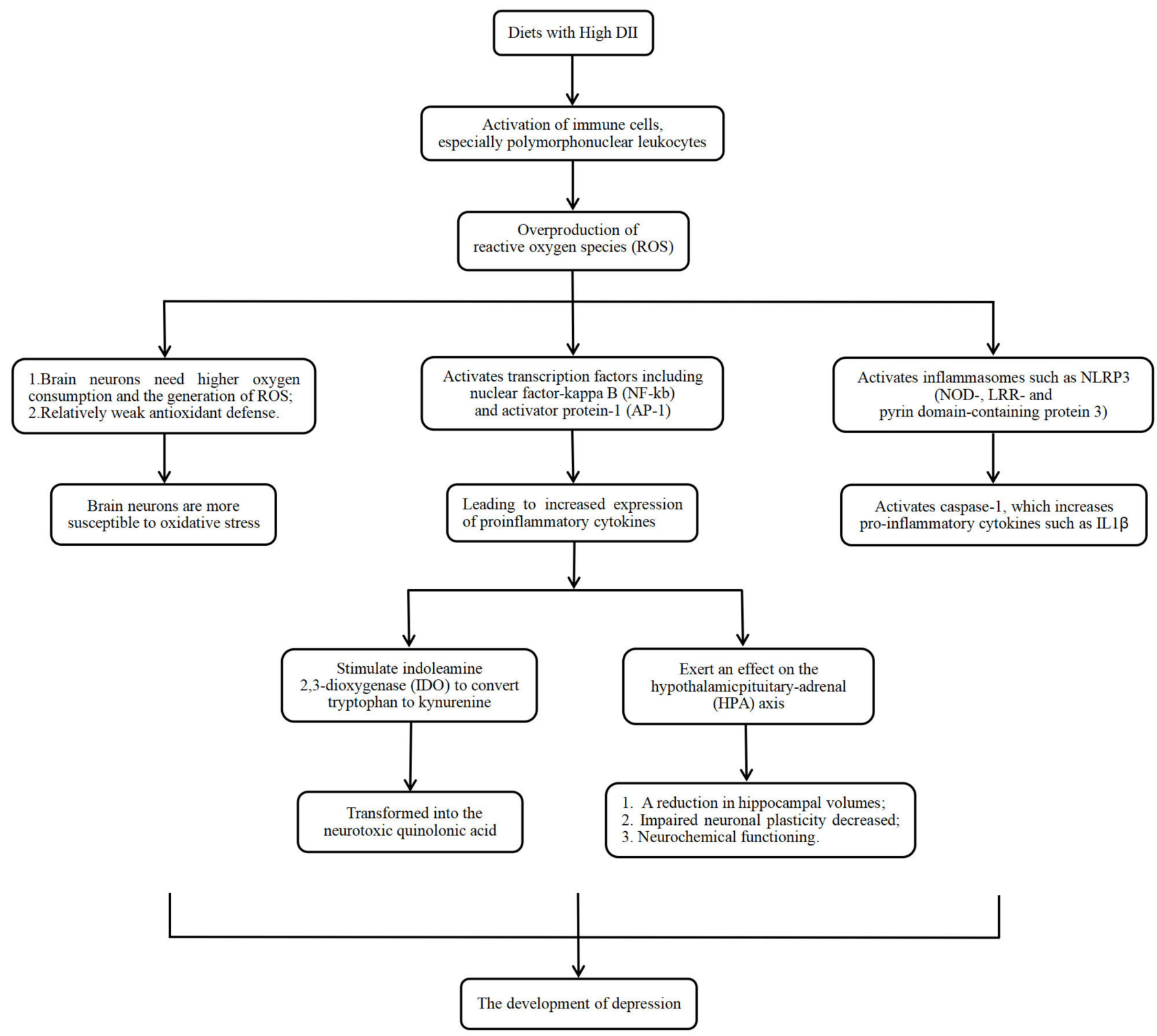

Figure I The possible main mechanism of dietary inflammatory index and depression. 
improved methods are needed to confirm the current findings.

\section{Funding}

Community Cohort Study on Specialized Nervous System Diseases (No.2017YFC0907701).

\section{Disclosure}

The authors report no conflicts of interest in this work.

\section{References}

1. Cancan H, Liang G, Yingying Y, et al. Amygdala connectivity mediates the association between anxiety and depression in patients with major depressive disorder. Brain Imaging Behav. 2019;13:1146-1159. doi:10.1007/s11682-018-9923-z

2. Wittenborn AK, Rahmandad H, Rick J, et al. Depression as a systemic syndrome: mapping the feedback loops of major depressive disorder. Psychol Med. 2016;46:551-562. doi:10.1017/S0033291715002044

3. Anderson Kevin M, Collins Meghan A, Kong R, et al. Convergent molecular, cellular, and cortical neuroimaging signatures of major depressive disorder. Proc Natl Acad Sci U S A. 2020;117:25138-25149. doi:10.1073/pnas.2008004117

4. Jonathan R, Marco M, Susanne M, et al. Severity of current depression and remission status are associated with structural connectome alterations in major depressive disorder. Mol Psychiatry. 2020;25:1550-1558. doi:10.1038/s41380-019-0603-1

5. Smith K. Mental health: a world of depression. Nature. 2014;515:181. doi:10.1038/515180a

6. Casey DA. Depression in older adults: a treatable medical condition. Prim Care. 2017;44:499-510. doi:10.1016/j. pop.2017.04.007

7. Stanners MN, Barton CA, Shakib S, et al. Depression diagnosis and treatment amongst multimorbid patients: a thematic analysis. BMC Fam Pract. 2014;15:124. doi:10.1186/1471-2296-15-124

8. Opie RS, O'Neil A, Itsiopoulos C, et al. The impact of whole-ofdiet interventions on depression and anxiety: a systematic review of randomised controlled trials. Public Health Nutr. 2015;18:2074-2093. doi:10.1017/S1368980014002614

9. Bremner JD, Moazzami K, Wittbrodt MT, et al. Diet, stress and mental health. Nutrients. 2020;12:2428. doi:10.3390/nu12082428

10. Dash S, Clarke G, Berk M, et al. The gut microbiome and diet in psychiatry: focus on depression. Curr Opin Psychiatry. 2015;28:1-6. doi:10.1097/YCO.0000000000000117

11. Molendijk M, Molero P, Ortuño Sánchez-Pedreño F, et al. Diet quality and depression risk: a systematic review and dose-response meta-analysis of prospective studies. J Affect Disord. 2018;226:346-354. doi:10.1016/j.jad.2017.09.022

12. Włodarczyk A, Cubała WJ. Mechanisms of action of the ketogenic diet in depression. Neurosci Biobehav Rev 2019;107:422-423. doi:10.1016/j.neubiorev.2019.09.038

13. Michael B, Williams Lana J, Jacka Felice N, et al. So depression is an inflammatory disease, but where does the inflammation come from? BMC Med. 2013;11:200. doi:10.1186/1741-7015-11-200

14. Margreet K, Sahar EA. MIDtrauma consortium, Depressed gut? The microbiota-diet-inflammation trialogue in depression. Curr Opin Psychiatry. 2017;30:369-377. doi:10.1097/ YCO.0000000000000350

15. Lopresti Adrian L, Hood Sean D, Drummond Peter D. A review of lifestyle factors that contribute to important pathways associated with major depression: diet, sleep and exercise. $J$ Affect Disord. 2013;148:12-27. doi:10.1016/j.jad.2013.01.014
16. Shabbir F, Patel A, Mattison C, et al. Effect of diet on serotonergic neurotransmission in depression. Neurochem Int. 2013;62:324-329. doi:10.1016/j.neuint.2012.12.014

17. Null G, Pennesi L. Diet and lifestyle intervention on chronic moderate to severe depression and anxiety and other chronic conditions. Complement Ther Clin Pract. 2017;29:189-193. doi:10.1016/j.ctcp.2017.09.007

18. Li Y, Lv MR, Wei YJ, et al. Dietary patterns and depression risk: a meta-analysis. Psychiatry Res. 2017;253:373-382. doi:10.1016/ j.psychres.2017.04.020

19. Guan YF, Huang GB, Xu MD, et al. Anti-depression effects of ketogenic diet are mediated via the restoration of microglial activation and neuronal excitability in the lateral habenula. Brain Behav Immun. 2020;88:748-762. doi:10.1016/j.bbi.2020.05.032

20. Matta J, Hoertel N, Kesse-Guyot E, et al. Diet and physical activity in the association between depression and metabolic syndrome: constances study. J Affect Disord. 2019;244:25-32. doi:10.1016/j.jad.2018.09.072

21. Steven B, Jane S, Murgatroyd Christopher A. Association of peripheral interleukin-6 with global cognitive decline in non-demented adults: a meta-analysis of prospective studies. Front Aging Neurosci. 2017;9:438. doi:10.3389/fnagi.2017.00438

22. Kenji S, Jesus M-M, Alberto B-S, et al. Effects of mindfulness-based interventions on biomarkers and low-grade inflammation in patients with psychiatric disorders: a meta-analytic review. Int J Mol Sci. 2020;21. doi:10.3390/ ijms 21072484

23. Hani ZN, Newman Michelle G. Increased inflammation predicts nine-year change in major depressive disorder diagnostic status. J Abnorm Psychol. 2021. doi:10.1037/abn0000716

24. Marlena C, Sheri M, Korczak Daphne J. Depression and inflammation among children and adolescents: a meta-analysis. $J$ Affect Disord. 2020;277:940-948. doi:10.1016/j.jad.2020.09.025

25. Marzieh M, Saunders Erika FH, Engeland Christopher G. Inflammation and the dimensions of depression: a review. Front Neuroendocrinol. 2020;56:100800. doi:10.1016/j. yfrne.2019.100800

26. Smith Kimberley J, Bonnie A, Lucie O, et al. The association between C-reactive protein, Interleukin-6 and depression among older adults in the community: a systematic review and meta-analysis. Exp Gerontol. 2018;102:109-132. doi:10.1016/j. exger.2017.12.005

27. Weerahandi H, Paul S, Quintiliani LM, et al. A mobile health coaching intervention for controlling hypertension: single-Arm Pilot Pre-Post Study. JMIR Form Res. 2020;4:e13989. doi: $10.2196 / 13989$

28. Clifford Astbury C, Penney TL, Adams J. Home-prepared food, dietary quality and socio-demographic factors: a cross-sectional analysis of the UK National Diet and nutrition survey 2008-16. Int J Behav Nutr Phys Act. 2019;16:82. doi:10.1186/s12966-019-0846-x

29. Sacks FM, Svetkey LP, Vollmer WM, et al. Effects on blood pressure of reduced dietary sodium and the Dietary Approaches to Stop Hypertension (DASH) diet. DASH-Sodium Collaborative Research Group. $N$ Engl J Med. 2001;344:3-10. doi:10.1056/ NEJM200101043440101

30. Widmer RJ, Flammer AJ, Lerman LO, et al. The Mediterranean diet, its components, and cardiovascular disease. Am J Med. 2015;128:229-238. doi:10.1016/j.amjmed.2014.10.014

31. Rensma SP, van Sloten TT, Houben A, et al. Microvascular dysfunction is associated with worse cognitive performance: the Maastricht Study. Hypertension. 2020;75:237-245. doi:10.1161/ HYPERTENSIONAHA.119.13023

32. Lin D, Wang L, Yan S, et al. The Role of oxidative stress in common risk factors and mechanisms of cardio-cerebrovascular ischemia and depression. Oxid Med Cell Longev. 2019;2019:2491927. doi:10.1155/2019/2491927 
33. Robaczewska J, Kędziora-Kornatowska K, Kucharski R, et al. Decreased expression of heme oxygenase is associated with depressive symptoms and may contribute to depressive and hypertensive comorbidity. Redox Rep. 2016;21:209-218. doi:10.1080/13510002.2015.1101889

34. Shimbo D, Chaplin W, Crossman D, et al. Role of depression and inflammation in incident coronary heart disease events. $\mathrm{Am}$ J Cardiol. 2005;96:1016-1021. doi:10.1016/j. amjcard.2005.05.064

35. Krebs-Smith SM, Pannucci TE, Subar AF, et al. Update of the Healthy Eating Index: HEI-2015. J Acad Nutr Diet. 2018;118:1591-1602. doi:10.1016/j.jand.2018.05.021

36. Saeede JN, Matin G, Pegah R, et al. A case-control study of Dietary Approaches to Stop Hypertension (DASH) diets, colorectal cancer and adenomas among Iranian population. $B M C$ Cancer. 2021;21:1050. doi:10.1186/s12885-021-08786-5

37. Ferguson Christine C, Knol Linda L, Ellis Amy C. Visceral adiposity index and its association with Dietary Approaches to Stop Hypertension (DASH) diet scores among older adults: national Health and Nutrition Examination Surveys 2011-2014. Clin Nutr. 2021;40:4085-4089. doi:10.1016/j.clnu.2021.02.008

38. Krasimira A, Liselot K, Egea RC. Dietary patterns and biomarkers of oxidative stress and inflammation: a systematic review of observational and intervention studies. Redox Biol. 2021;42:101869. doi:10.1016/j.redox.2021.101869

39. Sepideh S, Chitsazi Maryam J, Amin S-A. The effect of dietary approaches to stop hypertension (DASH) on serum inflammatory markers: a systematic review and meta-analysis of randomized trials. Clin Nutr. 2018;37:542-550. doi:10.1016/j. clnu.2017.02.018

40. Andreas N, Patrik H, Fawzi K. Adherence to DASH-Style Dietary Pattern Impacts on Adiponectin and Clustered Metabolic Risk in Older Women. Nutrients. 2019;11. doi:10.3390/nu11040805

41. Razieh P, Neda H-E, Mojgan M, et al. Effect of DASH diet on oxidative stress parameters: a systematic review and meta-analysis of randomized clinical trials. Diabetes Metab Syndr. 2020;14:2131-2138. doi:10.1016/j.dsx.2020.10.031

42. Mohsen RZ, Hosein TM, Fereshteh B, et al. The effects of DASH diet on weight loss and metabolic status in adults with non-alcoholic fatty liver disease: a randomized clinical trial. Liver Int. 2016;36:563-571. doi:10.1111/liv.12990

43. Zatollah A, Mansooreh S, Zohreh T, et al. A randomized controlled clinical trial investigating the effect of DASH diet on insulin resistance, inflammation, and oxidative stress in gestational diabetes. Nutrition. 2013;29:619-624. doi:10.1016/j. nut.2012.11.020

44. Schwingshackl L, Bogensberger B, Hoffmann G. Diet quality as assessed by the healthy eating index, alternate healthy eating index, dietary approaches to stop hypertension score, and health outcomes: an updated systematic review and Meta-Analysis of Cohort Studies. J Acad Nutr Diet. 2018;118:74-100. doi:10.1016/ j.jand.2017.08.024

45. Morze J, Danielewicz A, Hoffmann G, et al. Diet quality as assessed by the healthy eating index, alternate healthy eating index, dietary approaches to stop hypertension score, and health outcomes: a second update of a systematic review and Meta-Analysis of Cohort Studies. $J$ Acad Nutr Diet. 2020;120:1998-2031. doi:10.1016/j.jand.2020.08.076

46. Reedy J, Lerman JL, Krebs-Smith SM, et al. Evaluation of the healthy eating index-2015. $J$ Acad Nutr Diet. 2018;118:1622-1633. doi:10.1016/j.jand.2018.05.019

47. Wu PY, Lin MY, Tsai PS. Alternate healthy eating index and risk of depression: a meta-analysis and systematic review. Nutr Neurosci. 2020;23:101-109. doi:10.1080/1028415X. 2018.1477424
48. Owen L, Corfe B. The role of diet and nutrition on mental health and wellbeing. Proc Nutr Soc. 2017;76:425-426. doi:10.1017/ S0029665117001057

49. Marx W, Lane M, Hockey M, et al. Diet and depression: exploring the biological mechanisms of action. Mol Psychiatry. 2021;26:134-150. doi:10.1038/s41380-020-00925-x

50. Lassale C, Batty GD, Baghdadli A, et al. Healthy dietary indices and risk of depressive outcomes: a systematic review and meta-analysis of observational studies. Mol Psychiatry. 2019;24:965-986. doi:10.1038/s41380-018-0237-8

51. Taylor AM, Holscher HD. A review of dietary and microbial connections to depression, anxiety, and stress. Nutr Neurosci. 2020;23:237-250. doi:10.1080/1028415X.2018.1493808

52. Quirk SE, Williams LJ, O'Neil A, et al. The association between diet quality, dietary patterns and depression in adults: a systematic review. BMC Psychiatry. 2013;13:175. doi:10.1186/1471-244X13-175

53. Sofi F, Macchi C, Abbate R, et al. Mediterranean diet and health status: an updated meta-analysis and a proposal for a literature-based adherence score. Public Health Nutr. 2014;17:2769-2782. doi:10.1017/S1368980013003169

54. Valls-Pedret C, Sala-Vila A, Serra-Mir M, et al. Mediterranean Diet and Age-Related Cognitive Decline: a Randomized Clinical Trial. JAMA Intern Med. 2015;175:1094-1103. doi:10.1001/ jamainternmed.2015.1668

55. Lourida I, Soni M, Thompson-Coon J, et al. Mediterranean diet, cognitive function, and dementia: a systematic review. Epidemiology. 2013;24:479-489. doi:10.1097/EDE.0b013e 3182944410

56. Galbete C, Kröger J, Jannasch F, et al. Nordic diet, Mediterranean diet, and the risk of chronic diseases: the EPIC-Potsdam study. BMC Med. 2018;16:99. doi:10.1186/s12916-018-1082-y

57. Féart C, Samieri C, Rondeau V, et al. Adherence to a Mediterranean diet, cognitive decline, and risk of dementia. JAMA. 2009;302:638-648. doi:10.1001/jama.2009.1146

58. Parletta N, Zarnowiecki D, Cho J, et al. A Mediterranean-style dietary intervention supplemented with fish oil improves diet quality and mental health in people with depression: a randomized controlled trial (HELFIMED). Nutr Neurosci. 2019;22:474-487. doi:10.1080/1028415X.2017.1411320

59. García-Toro M, Vicens-Pons E, Gili M, et al. Obesity, metabolic syndrome and Mediterranean diet: impact on depression outcome. $J$ Affect Disord. 2016;194:105-108. doi:10.1016/j. jad.2015.12.064

60. Riddhi S, Nour M, Memet E, et al. Mediterranean diet components are linked to greater endothelial function and lower inflammation in a pilot study of ethnically diverse women. Nutr Res. 2020;75:77-84. doi:10.1016/j.nutres.2020.01.004

61. Arouca Aline B, Aline M, Jean D, et al. Interplay between the Mediterranean diet and C-reactive protein genetic polymorphisms towards inflammation in adolescents. Clin Nutr. 2020;39:1919-1926. doi:10.1016/j.clnu.2019.08.016

62. Bédard A, Lamarche B, Corneau L, Dodin S, Lemieux S. Sex differences in the impact of the Mediterranean diet on systemic inflammation. Nutr J. 2015;14:46. doi:10.1186/s12937-015-0035-y

63. Hornedo-Ortega R, Cerezo AB, de Pablos RM, et al. Phenolic Compounds Characteristic of the Mediterranean Diet in Mitigating Microglia-Mediated Neuroinflammation. Front Cell Neurosci. 2018;12:373. doi:10.3389/fncel.2018.00373

64. Shivappa N, Steck SE, Hurley TG, et al. Designing and developing a literature-derived, population-based dietary inflammatory index. Public Health Nutr. 2014;17:1689-1696. doi:10.1017/ S1368980013002115

65. Shivappa N, Hebert JR, Marcos A, et al. Association between dietary inflammatory index and inflammatory markers in the HELENA study. Mol Nutr Food Res. 2017;61:10. 
66. Qin Z, Chang K, Liao R, et al. Greater Dietary Inflammatory Potential Is Associated With Higher Likelihood of Abdominal Aortic Calcification. Front Cardiovasc Med. 2021;8:720834. doi:10.3389/fcvm.2021.720834

67. Bondonno NP, Blekkenhorst LC, Bird AL, et al. Dietary inflammatory index and the aging kidney in older women: a 10-year prospective cohort study. Eur J Nutr. 2020;59:3201-3211. doi:10.1007/s00394-019-02160-9

68. Salari-Moghaddam A, Keshteli AH, Afshar H, et al. Association between dietary inflammatory index and psychological profile in adults. Clin Nutr. 2019;38:2360-2368. doi:10.1016/j. clnu.2018.10.015

69. Ariya M, Shahraki HR, Farjam M, et al. Dietary inflammatory index and metabolic syndrome in Iranian population (Fasa Persian Cohort Study). Sci Rep. 2020;10:16762. doi:10.1038/ s41598-020-73844-0

70. Turner-McGrievy GM, Wirth MD, Shivappa N, et al. Randomization to plant-based dietary approaches leads to larger short-term improvements in Dietary Inflammatory Index scores and macronutrient intake compared with diets that contain meat. Nutr Res. 2015;35:97-106. doi:10.1016/j. nutres.2014.11.007

71. Turner-McGrievy GM, Wirth MD, Shivappa N, et al. Impact of a 12-month Inflammation Management Intervention on the Dietary Inflammatory Index, inflammation, and lipids. Clin Nutr ESPEN. 2019;30:42-51. doi:10.1016/j.clnesp.2019.02.008

72. Crimarco A, Turner-McGrievy GM, Wirth MD, et al. Baseline markers of inflammation, lipids, glucose, and Dietary Inflammatory Index scores do not differ between adults willing to participate in an intensive inflammation reduction intervention and those who do not. Nutr Health. 2019;25:9-19. doi:10.1177/ 0260106018800645

73. Shakya PR, Melaku YA, Shivappa N, et al. Dietary inflammatory index $\left(\mathrm{DII}^{\circledR}\right)$ and the risk of depression symptoms in adults. Clin Nutr. 2021;40:3631-3642. doi:10.1016/j.clnu.2020.12.031

74. Aslani Z, Abshirini M, Heidari-Beni M, et al. Dietary inflammatory index and dietary energy density are associated with menopausal symptoms in postmenopausal women: a cross-sectional study. Menopause. 2020;27:568-578. doi:10.1097/ GME.0000000000001502

75. Wang T, Jiang $\mathrm{H}, \mathrm{Wu} \mathrm{Y}$, et al. The association between Dietary Inflammatory Index and disability in older adults. Clin Nutr. 2021;40:2285-2292. doi:10.1016/j.clnu.2020.10.017

76. Haghighatdoost F, Feizi A, Esmaillzadeh A, et al. Association between the dietary inflammatory index and common mental health disorders profile scores. Clin Nutr. 2019;38:1643-1650. doi:10.1016/j.clnu.2018.08.016

77. Almeida-de-souza J, Santos R, Barros R, et al. Dietary inflammatory index and inflammatory biomarkers in adolescents from LabMed physical activity study. Eur $J$ Clin Nutr. 2018;2:710-719.

78. Ma W, Jovani M, Nguyen LH, et al. Association Between Inflammatory Diets, Circulating Markers of Inflammation, and Risk of Diverticulitis. Clin Gastroenterol Hepatol. 2020;18:2279-2286. doi:10.1016/j.cgh.2019.11.011

79. Mickle AT, Brenner DR, Beattie $\mathrm{T}$, et al. The Dietary Inflammatory Index ${ }^{\circledR}$ and Alternative Healthy Eating Index 2010 in relation to leucocyte telomere length in postmenopausal women: a cross-sectional study. J Nutr Sci. 2019;8:e35. doi:10.1017/jns.2019.32

80. Laouali N, Mancini FR, Hajji-Louati M, et al. Dietary inflammatory index and type 2 diabetes risk in a prospective cohort of 70,991 women followed for 20 years: the mediating role of BMI. Diabetologia. 2019;62:2222-2232. doi:10.1007/s00125-01904972-0
81. Ruiz-Canela M, Zazpe I, Shivappa N, et al. Dietary inflammatory index and anthropometric measures of obesity in a population sample at high cardiovascular risk from the PREDIMED (PREvención con DIeta MEDiterránea) trial. $\mathrm{Br} \quad \mathrm{J}$ Nutr. 2015;113:984-995. doi:10.1017/S0007114514004401

82. MacDonald CJ, Laouali N, Madika AL, et al. Dietary inflammatory index, risk of incident hypertension, and effect modification from BMI. Nutr J. 2020;19:62. doi:10.1186/s12937-020-00577-1

83. Ghorabi S, Esteghamati A, Azam K, et al. Association between dietary inflammatory index and components of metabolic syndrome. $J$ Cardiovasc Thorac Res. 2020;12:27-34. doi: $10.34172 /$ jevtr.2020.05

84. Oddy WH, Allen KL, Trapp GSA, et al. Dietary patterns, body mass index and inflammation: pathways to depression and mental health problems in adolescents. Brain Behav Immun. 2018;69:428-439. doi:10.1016/j.bbi.2018.01.002

85. Navarro P, Shivappa N, Hébert JR, et al. Predictors of the dietary inflammatory index in children and associations with childhood weight status: a longitudinal analysis in the Lifeways Cross-Generation Cohort Study. Clin Nutr. 2020;39:2169-2179. doi:10.1016/j.clnu.2019.09.004

86. King DE, Xiang J. The Dietary Inflammatory Index Is Associated With Diabetes Severity. J Am Board Fam Med. 2019;32:801-806. doi:10.3122/jabfm.2019.06.190092

87. King DE, Xiang J. The Dietary Inflammatory Index Is Associated With Diabetes Severity. J Am Board Fam Med. 2019;32:801-806.

88. Muhammad HFL, van Baak MA, Mariman EC, et al. Dietary Inflammatory Index Score and Its Association with Body Weight, Blood Pressure, Lipid Profile, and Leptin in Indonesian Adults. Nutrients. 2019;11:148. doi:10.3390/nu11010148

89. Frühbeck G, Toplak H, Woodward E, Yumuk V, Maislos M, Oppert JM. Executive Committee of the European Association for the Study of Obesity. Obesity: the gateway to ill health - an EASO position statement on a rising public health, clinical and scientific challenge in Europe. Obes Facts. 2013;6:117-120. doi:10.1159/000350627

90. Fernández-Sánchez A, Madrigal-Santillán E, Bautista M, et al. Inflammation, oxidative stress, and obesity. Int $J$ Mol Sci. 2011;12:3117-3132. doi:10.3390/ijms 12053117

91. Kotzbeck P, Giordano A, Mondini E, et al. Brown adipose tissue whitening leads to brown adipocyte death and adipose tissue inflammation. J Lipid Res. 2018;59:784-794. doi:10.1194/jlr. M079665

92. Alina S, Dan D, Philippe C, et al. Inflammation, a link between obesity and atrial fibrillation. Inflamm Res. 2015;64:383-393. doi:10.1007/s00011-015-0827-8

93. Katja B, Julius W, Ryan C, et al. Body Mass Index (BMI) and Its Influence on the Cardiovascular and Operative Risk Profile in Coronary Artery Bypass Grafting Patients: impact of Inflammation and Leptin. Oxid Med Cell Longev. 2020;2020:5724024. doi:10.1155/2020/5724024

94. Shah R, Lu Y, Hinkle CC, et al. Gene profiling of human adipose tissue during evoked inflammation in vivo. Diabetes. 2009;58:2211-2219. doi:10.2337/db09-0256

95. Karimbeiki R, Alipoor E, Yaseri M, et al. Association between the dietary inflammatory index and obesity in otherwise healthy adults: role of age and sex. Int J Clin Pract. 2021;75:e14567. doi:10.1111/ijcp. 14567

96. Navarro P, Shivappa N, Hébert JR, et al. Intergenerational associations of dietary inflammatory index with birth outcomes and weight status at age 5 and 9: results from the Lifeways cross-generation cohort study. Pediatr Obes. 2020;15:e12588. doi:10.1111/ijpo.12588

97. Chen X, Maguire B, Brodaty H, et al. Dietary Patterns and Cognitive Health in Older Adults: a Systematic Review. J Alzheimers Dis. 2019;67:583-619. doi:10.3233/JAD-180468 
98. Kerr J, Anderson C, Lippman SM. Physical activity, sedentary behaviour, diet, and cancer: an update and emerging new evidence. Lancet Oncol. 2017;18:e457-e471. doi:10.1016/ S1470-2045(17)30411-4

99. Accardi G, Shivappa N, Di Maso M, et al. Dietary inflammatory index and cancer risk in the elderly: a pooled-analysis of Italian case-control studies. Nutrition. 2019;63-64:205-210. doi:10.1016/j.nut.2019.02.008

100. Cervo MM, Shivappa N, Hebert JR, et al. Longitudinal associations between dietary inflammatory index and musculoskeletal health in community-dwelling older adults. Clin Nutr. 2020;39:516-523. doi:10.1016/j.clnu.2019.02.031

101. Hart MJ, Torres SJ, McNaughton SA, et al. A Dietary Inflammatory Index and associations with C-reactive protein in a general adult population. Eur J Nutr. 2021;60(7):4093-4106. doi:10.1007/s00394-021-02573-5

102. Beurel E, Toups M, Nemeroff CB. The Bidirectional Relationship of Depression and Inflammation: double Trouble. Neuron. 2020;107:234-256. doi:10.1016/j.neuron.2020.06.002

103. Kohler O, Krogh J, Mors O, et al. Inflammation in Depression and the Potential for Anti-Inflammatory Treatment. Curr Neuropharmacol. 2016;14:732-742. doi:10.2174/ 1570159X14666151208113700

104. Kiecolt-Glaser JK, Derry HM, Fagundes CP. Inflammation: depression fans the flames and feasts on the heat. Am $J \quad$ Psychiatry. 2015;172:1075-1091. doi:10.1176/appi. ajp.2015.15020152

105. Zhang JC, Yao W, Brain-derived Neurotrophic HK. Factor (BDNF)-TrkB Signaling in Inflammation-related Depression and Potential Therapeutic Targets. Curr Neuropharmacol. 2016;14:721-731. doi:10.2174/1570159X14666160119094646

106. Leonard BE. Inflammation and depression: a causal or coincidental link to the pathophysiology? Acta Neuropsychiatr. 2018;30:1-16. doi:10.1017/neu.2016.69

107. Halaris A. Inflammation-Associated Co-morbidity Between Depression and Cardiovascular Disease. Curr Top Behav Neurosci. 2017;31:45-70.

108. Miller AH, Maletic V. Inflammation and its discontents: the role of cytokines in the pathophysiology of major depression. Biol Psychiatry. 2009;65:732-741. doi:10.1016/j. biopsych.2008.11.029

109. Halaris A. Inflammation and depression but where does the inflammation come from? Curr Opin Psychiatry. 2019;32:422-428. doi:10.1097/YCO.0000000000000531

110. D’Mello C, Swain MG. Immune-to-Brain Communication Pathways in Inflammation-Associated Sickness and Depression. Curr Top Behav Neurosci. 2017;31:73-94.

111. Leff-Gelman P, Mancilla-Herrera I, Flores-Ramos M, et al. The Immune System and the Role of Inflammation in Perinatal Depression. Neurosci Bull. 2016;32:398-420. doi:10.1007/ s12264-016-0048-3

112. Felger JC, Haroon E, Patel TA, et al. What does plasma CRP tell us about peripheral and central inflammation in depression? Mol Psychiatry. 2020;25:1301-1311. doi:10.1038/s41380-018-0096-3

113. Malcomson FC, Willis ND, McCallum I, et al. Diet-Associated Inflammation Modulates Inflammation and WNT Signaling in the Rectal Mucosa, and the Response to Supplementation with Dietary Fiber. Cancer Prev Res. 2021;14:337-346. doi:10.1158/ 1940-6207.CAPR-20-0335

114. Burrows K, Stewart JL, Antonacci C, et al. Association of poorer dietary quality and higher dietary inflammation with greater symptom severity in depressed individuals with appetite loss. J Affect Disord. 2020;263:99-106. doi:10.1016/j.jad.2019.11.160
115. Shivappa N, Wirth MD, Murphy EA, et al. Association between the Dietary Inflammatory Index (DII) and urinary enterolignans and C-reactive protein from the National Health and Nutrition Examination Survey-2003-2008. Eur J Nutr. 2019;58:797-805. doi:10.1007/s00394-018-1690-5

116. Ruiz-Saavedra S, Salazar N, Suárez A, et al. Comparison of Different Dietary Indices as Predictors of Inflammation, Oxidative Stress and Intestinal Microbiota in Middle-Aged and Elderly Subjects. Nutrients. 2020;12(12):3828. doi:10.3390/ nu12123828

117. Ghazizadeh H, Yaghooti-Khorasani M, Asadi Z, et al. Association between Dietary Inflammatory Index $\left(\mathrm{DII}^{\mathbb{R}}\right)$ and depression and anxiety in the Mashhad Stroke and Heart Atherosclerotic Disorder (MASHAD) Study population. BMC Psychiatry. 2020;20:282. doi:10.1186/s12888-020-02663-4

118. Wirth MD, Shivappa N, Burch JB, et al. The Dietary Inflammatory Index, shift work, and depression: results from NHANES. Health Psychol. 2017;36:760-769. doi:10.1037/ hea0000514

119. Sánchez-Villegas A, Ruíz-Canela M, de la Fuente-arrillaga C, et al. Dietary inflammatory index, cardiometabolic conditions and depression in the Seguimiento Universidad de Navarra cohort study. $\quad B r \quad J \quad N u t r . \quad 2015 ; 114: 1471-1479 . \quad$ doi:10.1017/ S0007114515003074

120. Jorgensen D, White GE, Sekikawa A, et al. Higher dietary inflammation is associated with increased odds of depression independent of Framingham Risk Score in the National Health and Nutrition Examination Survey. Nutr Res. 2018;54:23-32. doi:10.1016/j.nutres.2018.03.004

121. Attwells S, Setiawan E, Wilson AA, et al. Inflammation in the Neurocircuitry of Obsessive-Compulsive Disorder. JAMA Psychiatry. 2017;74:833-840. doi:10.1001/jamapsychiatry. 2017.1567

122. Bergmans RS, Malecki KM. The association of dietary inflammatory potential with depression and mental well-being among U. S. Adults Prev Med. 2017;99:313-319. doi:10.1016/j. ypmed.2017.03.016

123. Hariri N, Darafshi Ghahroudi S, Jahangiri S, et al. The beneficial effects of sumac (Rhus coriaria L.) supplementation along with restricted calorie diet on anthropometric indices, oxidative stress, and inflammation in overweight or obese women with depression: a randomized clinical trial. Phytother Res. 2020;34:3041-3051. doi:10.1002/ptr.6737

124. Akbaraly T, Kerlau C, Wyart M, et al. Dietary inflammatory index and recurrence of depressive symptoms: results from the Whitehall II Study. Clin Psychol Sci. 2016;4:1125-1134. doi:10.1177/2167702616645777

125. Phillips CM, Shivappa N, Hébert JR, et al. Dietary inflammatory index and mental health: a cross-sectional analysis of the relationship with depressive symptoms, anxiety and well-being in adults. Clin Nutr. 2018;37:1485-1491. doi:10.1016/j.clnu.2017.08.029

126. Gomes AP, Gonçalves H, Dos santos Vaz J, et al. Do inflammation and adiposity mediate the association of diet quality with depression and anxiety in young adults? Clin Nutr. 2021;40:2800-2808. doi:10.1016/j.clnu.2021.03.028 


\section{Publish your work in this journal}

The Journal of Inflammation Research is an international, peerreviewed open-access journal that welcomes laboratory and clinical findings on the molecular basis, cell biology and pharmacology of inflammation including original research, reviews, symposium reports, hypothesis formation and commentaries on: acute/chronic inflammation; mediators of inflammation; cellular processes; molecular mechanisms; pharmacology and novel anti-inflammatory drugs; clinical conditions involving inflammation. The manuscript management system is completely online and includes a very quick and fair peerreview system. Visit http://www.dovepress.com/testimonials.php to read real quotes from published authors. 